\title{
Efeitos laxativos do polietilenoglicol 3350 e de soluções eletrolíticas em equinos
}

\author{
[Laxative effects of polyethylene glycol 3350 and electrolyte solutions in equines] \\ C.L.N. Gomes ${ }^{1}$, J.D. Ribeiro Filho ${ }^{2}$ \\ ${ }^{1}$ Universidade Estadual do Maranhão - São Luís, MA \\ ${ }^{2}$ Universidade Federal de Viçosa - Viçosa, MG
}

\begin{abstract}
RESUMO
Compararam-se a motilidade intestinal, a frequência de defecação, a umidade e o aspecto das fezes, o tempo médio de retenção (TMR) e a taxa de passagem (TxP) da ingesta no intestino grosso (ig), em cinco éguas hígidas tratadas com: polietilenoglicol 3350, na dose única de $1,5 \mathrm{~g} \mathrm{~kg}^{-1}$, em bolus, por via enteral (PEG); ou por polietilenoglicol 3350, na dose única de $1,5 \mathrm{~g} \mathrm{~kg}^{-1}$, em bolus, por via enteral, associado ao Ringer lactato, $15 \mathrm{~mL} \mathrm{~kg}^{-1} \mathrm{~h}^{-1}$ por fluxo contínuo de $12 \mathrm{~h}$, via intravenosa (PEG+RL); ou por solução isotônica poliônica enteral, $15 \mathrm{~mL} \mathrm{~kg}^{-1} \mathrm{~h}^{-1}$ em fluxo contínuo de $12 \mathrm{~h}$ (SIPE); ou por solução isotônica poliônica enteral, $7,5 \mathrm{~mL} \mathrm{~kg}^{-1} \mathrm{~h}^{-1}$ por fluxo contínuo de $12 \mathrm{~h}$, via enteral, associada ao Ringer lactato, $7,5 \mathrm{~mL} \mathrm{~kg}^{-1} \mathrm{~h}^{-1}$ por fluxo contínuo de $12 \mathrm{~h}$, via intravenosa (SIPE+RL); ou por cloreto de sódio $0,9 \%$, $15 \mathrm{~mL} \mathrm{~kg}^{-1} \mathrm{~h}^{-1}$ por fluxo contínuo de $12 \mathrm{~h}$, via intravenosa ( $\left.\mathrm{NaCl} 0,9 \%\right)$. O PEG apenas amoleceu discretamente as fezes. O PEG+RL, o SIPE+RL e o $\mathrm{NaCl} 0,9 \%$ foram mais eficazes em amolecer as fezes e aumentar a frequência de defecação do que o PEG. O SIPE foi o único tratamento que aumentou a motilidade intestinal, e foi o melhor em aumentar a frequência de defecações, amolecer as fezes e diminuir o TMRig, aumentando a TxPig. Conclui-se que os cinco tratamentos demonstraram efeito laxativo, sendo o SIPE o mais eficaz.
\end{abstract}

Palavras-chaves: equinos, laxativos, polietilenoglicol, soluções

\begin{abstract}
The intestinal motility, frequency of defecation, moisture and appearance of feces, mean retention time $(M R T)$ and passage rate (RP) of ingesta in the large intestine (li) were compared in five healthy mares treated with: polyethylene glycol 3350 in a single dose of $1.5 \mathrm{~g} \mathrm{~kg}^{-1}$, in bolus, administered enterally (PEG); or for polyethylene glycol 3350 in a single dose of $1.5 \mathrm{~g} \mathrm{~kg}^{-1}$, in bolus, enterally, associated to the

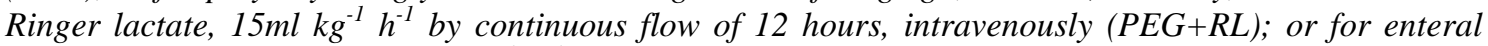

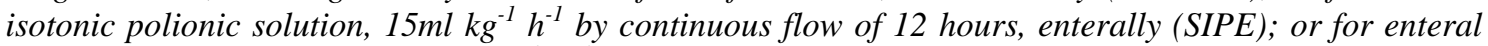

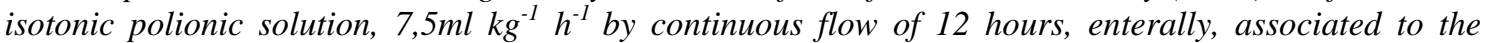
Ringer lactate, $7.5 \mathrm{~mL} \mathrm{~kg}^{-1} \mathrm{~h}^{-1}$ by continuous flow of 12 hours, intravenously (SIPE $\left.+R L\right)$; or sodium chloride $0.9 \%, 15 \mathrm{ml} \mathrm{kg^{-1 }} \mathrm{h}^{-1}$ by continuous flow of 12 hours, intravenously (NaCl 0.9\%). PEG treatment only slightly softened feces. PEG+RL, SIPE $+R L$ and NaCl $0.9 \%$ were more effective in softening the feces and increasing the frequency of defecation than PEG. SIPE was the best to increase the frequency of defecation, to soften the feces, and reduce the TMRli, increasing RPli. It is concluded that the five treatments demonstrated a laxative effect, but SIPE was the most effective.
\end{abstract}

Keywords: equines, laxatives, polyethylene glycol, solutions

Recebido em 14 de março de 2012

Aceito em 1 de agosto de 2012

E-mail: claudioninagomes@uol.com.br 


\section{INTRODUÇÃO}

Nos equinos, o trânsito intestinal é comumente avaliado por meio de auscultação dos movimentos intestinais, frequência de defecação, aspecto e teor de umidade das fezes. Esses parâmetros são de grande importância quando os animais estão acometidos por cólica.

Entre as doenças causadoras de cólica em equinos, a compactação do intestino grosso é uma das mais frequentes (Dabareiner e White, 1995) e se caracteriza por uma formação firme de ingesta ressecada no lúmen do ceco ou dos cólons, retardando o trânsito intestinal (Thomassian, 2005).

Alguns equinos com compactação respondem eficazmente a tratamentos com analgésicos, laxativos e hidratação enteral ou intravenosa (Sullins, 1990). Alguns estudos avaliaram os efeitos destas terapias em equinos com compactação experimental no intestino grosso, avaliando o tempo médio de retenção e a taxa de passagem da ingesta no trato digestivo (Macoris, 1989; Lopes, 2002; Ribeiro Filho, 2003).

Laxativos são compostos que aumentam a frequência da defecação e o volume fecal, ou alteram a consistência fecal (Clark e Becht, 1987). Um laxativo osmótico cujo princípio ativo é o polietilenoglicol (PEG 3350) é efetivo no tratamento da constipação e na lavagem intestinal para procedimentos diagnósticos e cirurgias intestinais em humanos (Corazziari et al., 1996; Hammer et al., 1989). O efeito laxativo do PEG 3350 em equinos ainda não é conhecido.

A hidratação por via enteral estimula a motilidade intestinal e aumenta o teor de água nas fezes e a frequência de defecação, portanto exerce efeito laxativo. Como nos equinos o trânsito de fluidos do estômago até o intestino grosso é rápido (Argênzio et al., 1974), a ingesta é hidratada rapidamente após a infusão enteral (Lopes, 2002).

O Ringer lactato e o cloreto de sódio $(\mathrm{NaCl})$ $0,9 \%$ são soluções para uso intravenoso (IV) utilizadas para recompor o equilíbrio hidroeletrolítico e o ácido-base. Efeitos do Ringer lactato IV e do Ringer lactato IV associado a laxativo por via enteral sobre a motilidade intestinal de equinos foram averiguados (Lopes, 2002; Ribeiro Filho, 2003), porém não há relato quanto à infusão intravenosa de $\mathrm{NaCl} 0,9 \%$.

Este estudo teve o objetivo de avaliar e comparar os efeitos laxativos de alguns tratamentos utilizando o PEG 3350 e soluções eletrolíticas administradas por via enteral ou intravenosa sobre motilidade intestinal, frequência de defecação, umidade e aspecto das fezes, tempo médio de retenção e taxa de passagem da ingesta no intestino grosso de equinos hígidos.

\section{MATERIAL E MÉTODOS}

Foram utilizadas cinco éguas hígidas (uma da raça Mangalarga Marchador, duas 1/2 sangue da raça Mangalarga Marchador e duas 7/8 da raça Bretã), não gestantes, com idades entre cinco e 19 anos (média de idade de 12,5 anos), com bons escores corporais, peso corporal médio de 392,0 quilogramas $(\mathrm{kg})$, sem evidência de doenças (com base em exame físico, hemograma, exame bioquímico e urinálise), e sem histórico de doença intestinal nos últimos seis meses antes do início do estudo.

Dez dias antes do início do experimento os animais foram submetidos a exames clínicos e foram vermifugados com ivermectina associada à praziquantel (Padock NF gel pasta ${ }^{\circledR}-$ Laboratório Vetbrands ) por via oral e receberam banho de aspersão com carrapaticida a base de deltrametrina $0,025 \%$ (Butox $^{\odot}$ - Laboratório Quimio). Em seguida, foram alojados em baias individuais para adaptação à dieta constituída de feno de Tifton 85 e concentrado (Ração Equisul 15 especial - Total Alimentos) que foram fornecidos em duas refeições ao dia (seis e 18 horas), simultaneamente, e em partes iguais em cada refeição, adotando-se o consumo diário individual de $2 \%$ do peso corporal em matéria seca, sendo $40 \%$ de concentrado e $60 \%$ de volumoso. Ao concentrado fornecido pela manhã foi associado $50 \mathrm{~g} / \mathrm{dia}$ de suplemento mineral (Hiposal 80\% - Total Alimentos). Água foi fornecida "ad libitum" em bebedouros individuais.

Realizou-se um revezamento de tratamento/ animal/período, constituindo um delineamento cross-over 5X5: em cada período, um animal recebeu, randomicamente, um tratamento 
diferente dos administrados concomitantemente aos demais, totalizando cinco amostras/ tratamento $(n=5)$ no final do quinto e último período. Para não ocorrer interferência de efeito de um tratamento sobre o do período subsequente num mesmo animal, preconizou-se um intervalo de 14 dias entre os períodos.

Os tratamentos foram: polietilenoglicol 3350 (PEG): $1,5 \mathrm{~g} \mathrm{~kg}^{-1}$ diluído em cinco litros de água, fornecido via enteral (VE) por sonda nasogástrica (sonda nasogástrica n.11 - Provar), uma única dose em bolus. Cada sachê do produto comercial (Muvinlax $^{\odot}$ - Libbs) continha: 13,1250g de PEG 3350; 0,3507g de $\mathrm{NaCl}$; $0,0460 \mathrm{~g}$ de $\mathrm{KCl} ; 0,1775 \mathrm{~g}$ de $\mathrm{NaHCO}_{3}$. A osmolaridade do PEG foi de 252,66mMol, e seu $\mathrm{pH}$ foi 8,65; polietilenoglicol 3350, VE, associado ao Ringer lactato (solução de Ringer com lactato de sódio - Sanobiol), IV (PEG+RL). O polietilenoglicol 3350 foi administrado na mesma posologia do tratamento anterior. Imediatamente após a administração deste, foi administrada a solução de Ringer lactato, na dose de $15 \mathrm{~mL} \mathrm{~kg} \mathrm{~h}^{-1} \mathrm{~h}^{-1}$, IV, durante 12 horas. Osmolaridade do Ringer lactato: $272,22 \mathrm{mMol} \mathrm{L}^{-}$ 1, pH: 6,75; solução isotônica poliônica enteral (SIPE): $15 \mathrm{~mL} \mathrm{~kg}^{-1} \mathrm{~h}^{-1}$ durante 12 horas sob fluxo contínuo, por sonda nasoesofágica de pequeno calibre (4mm). Composição da solução: $6 \mathrm{~g}$ de $\mathrm{NaCl} ; 0,5 \mathrm{~g}$ de $\mathrm{KCl} ; 1 \mathrm{~g}$ de gluconato de cálcio; $0,3 \mathrm{~g}$ de pidolato de magnésio; $5 \mathrm{~g}$ de maltodextrina, diluídos em $1.000 \mathrm{~mL}$ de água. Osmolaridade da solução: $321,14 \mathrm{mMol} \mathrm{L}^{-1}$; pH: 7,20; solução isotônica poliônica enteral associada ao Ringer lactato (SIPE+RL). A SIPE foi administrada por via enteral, na dose de $7,5 \mathrm{~mL} \mathrm{~kg} \mathrm{~h}^{-1}$ durante 12 horas em fluxo contínuo, por sonda nasoesofágica de pequeno calibre (sonda uretral para cavalos - Provar). Concomitantemente, administrou-se o Ringer lactato na dose de $7,5 \mathrm{~mL} \mathrm{~kg}^{-1} \mathrm{~h}^{-1}$, IV, durante 12 horas. A composição da solução isotônica poliônica enteral foi a mesma utilizada no tratamento SIPE, e a do Ringer lactato a mesma do tratamento PEG+RL; solução de cloreto de sódio a $0,9 \%(\mathrm{NaCl} \quad 0,9 \%): 15 \mathrm{~mL} \mathrm{~kg}^{-1} \mathrm{~h}^{-1}$, durante 12 horas, por via intravenosa. Osmolaridade da solução: $309,80 \mathrm{mMol} \mathrm{L}^{-1}$; $\mathrm{pH}$ : 5,60 .

Os animais foram mantidos em jejum hídricoalimentar até o final dos tratamentos. Os movimentos intestinais, a umidade e o aspecto das fezes foram avaliados nos seguintes tempos: pré-tratamento (T0h: antes do início do tratamento); transtratamento (T6h: após seis horas de tratamento realizado; T12h: após doze horas de tratamento, que correspondeu ao tempo final de tratamento); pós-tratamento (T24h: doze horas após o término do tratamento, ou seja, ao T12h; T48h: 36 horas após o término do tratamento).

A motilidade intestinal foi auscultada com auxílio de estetoscópio modelo Rappaport, avaliando-se os borborigmos intestinais em quatro quadrantes abdominais: dorsal e ventral do lado direito do abdômen (que correspondiam à região de auscultação da base e do corpo-ápice do ceco, respectivamente); dorsal e ventral do lado esquerdo do abdômen (que correspondiam à auscultação do cólon dorsal e ventral esquerdo, respectivamente). A classificação da motilidade e seus respectivos escores foram: ausente $=0$; diminuída $=1 ;$ sem alteração $($ normal $)=2$; aumentada $=3$.

Quanto ao aspecto, as fezes foram classificadas segundo os seguintes escores: ressecadas $=0$ (em formato de bolota, ressecadas, com presença ou não de muco; normais = 1 (em formato de bolota e com consistência normal); pastosas $=2$ (na forma de pasta); aquosas ou diarreicas $=3$ (na forma de líquido).

Foram colhidas fezes do reto dos animais para ser obtido o percentual de umidade da seguinte maneira: foram pesadas $150 \mathrm{~g}$ de fezes, o que foi considerado como peso pré-secagem. Após pesadas, foram mantidas em estufa a $60^{\circ} \mathrm{C}$ por $72 \mathrm{~h}$ e, então, novamente pesadas para ser obtido o peso pós-secagem. O percentual (\%) de umidade das fezes (UF) foi obtido, segundo Avanza (2007), pela fórmula:

$\mathrm{UF}(\%)=[($ peso pré-secagem - peso póssecagem)/peso pré-secagem] x 100.

A frequência das defecações foi realizada em oito tempos: 0-6h, 6-12h, 12-18h, 18-24h, 2430h, 30-36h, 36-42h e 42-48h. Durante esses períodos, foi registrado o número de defecação dos animais.

Para se avaliar o efeito dos tratamentos sobre o trânsito intestinal, preparou-se do feno de Tifton 85 fornecido aos animais durante o experimento uma fração contendo um indicador externo, o 
cromomordente, produzido conforme Úden et al. (1980). Cinco horas antes do início dos tratamentos, os animais ingeriram $250 \mathrm{~g}$ de feno contendo o indicador cromomordente. Foram coletadas aproximadamente $300 \mathrm{~g}$ de fezes da ampola retal dos animais, nos seguintes momentos: zero (imediatamente antes do início dos tratamentos); três, seis, 12 (quando os animais receberam os tratamentos, portanto, no trans-tratamento); 18, 24, 30, 36, 48, 72, 96, 120 e 144 (quando os animais não mais recebiam os tratamentos). Imediatamente após cada coleta, colocaram-se as fezes em sacos plásticos e estes foram devidamente identificados. Em seguida, foram acondicionados em temperatura de $-15^{\circ} \mathrm{C}$ até o início da análise do cromo por absorção atômica (Aparelho de absorção atômica, GBC, modelo Avanta ${ }^{\Sigma}$ - Analítica).

Concluída a análise de cromo em cada amostra de fezes, o trânsito intestinal foi estimado por intermédio do ajustamento à curva de excreção fecal do indicador do modelo $Г(2)$ tempodependente descrito por Ellis et al. (1994), obtida por meio da fórmula: $\mathrm{C}_{\mathrm{t}}=\mathrm{Z} \times(\mathrm{t}-\tau) \times \gamma \times \operatorname{esp}[-$ $\gamma \times(\mathrm{t}-\tau)]$, sendo: $\mathrm{C}_{\mathrm{t}}=$ concentração fecal do indicador no tempo " $\mathrm{t}$ " ( $\mathrm{ppm}) ; \mathrm{t}=$ tempo após o fornecimento do indicador $(h) ; \gamma=$ parâmetro taxa-dependente relativo ao fluxo de partículas fibrosas no intestino grosso $\left(\mathrm{h}^{-1}\right) ; \mathrm{Z}=$ parâmetro sem interpretação biológica direta (ppm x h); $\tau=$ tempo decorrido entre a aplicação e o aparecimento do indicador nas fezes (h).

A taxa de passagem da fibra (TxP) e o tempo médio de retenção (TMR) pelo intestino grosso (ig) foram estimados pelas seguintes equações, segundo Ellis et al. (1994): TxPig $\left(\% \mathrm{~h}^{-1}\right)=2 / \gamma$; TMRig $(\mathrm{h})=$ TMRig $+\tau$.

Para análise estatística, submeteram-se as variáveis à ANOVA para verificar os efeitos do tratamento em função do tempo e do tempo em função do tratamento para as variáveis teor de umidade das fezes, TxPig e TMRig. Adotou-se o delineamento em quadrado latino, padronizandoo em $5 \times 5$ e considerando os efeitos de período, tratamento e tempo, sendo o efeito animal como aleatório. As variáveis foram analisadas no programa estatístico SAEG (Sistema..., 2007). Quando houve significância pelo teste F, as médias foram comparadas pelo teste de Tukey, sendo adotado $\mathrm{P}<0,05$. A mediana foi utilizada para avaliar variáveis classificadas em escores.

Esta pesquisa foi aprovada pelo Comitê de Ética para Uso de Animais do Departamento de Medicina Veterinária da Universidade Federal de Viçosa, protocolo n.050/2008.

\section{RESULTADOS E DISCUSSÃO}

O jejum hídrico-alimentar durante o TOh e o $\mathrm{T} 12 \mathrm{~h}$ possivelmente contribuiu com a hipomotilidade intestinal detectada em todos os quadrantes no grupo PEG (T6h e T12h), e nos quadrantes dorsal e ventral direito no $\mathrm{NaCl} 0,9 \%$ (T6h), pois, com o retorno da dieta aos animais, a motilidade intestinal foi normalizada (Tab. 1).

Como não houve hipomotilidade intestinal nos tratamentos PEG+RL, SIPE e SIPE+RL no referido período (Tab. 1), é possível que o cálcio contido nas soluções administradas nesses grupos tenha contribuído para a manutenção da motilidade intestinal, principalmente o gluconato de cálcio, nos grupos em que foi utilizada a solução enteral, pois, no SIPE, ocorreu aumento da motilidade intestinal no $\mathrm{T} 12 \mathrm{~h}$ nos quadrantes dorsal e ventral do abdômen esquerdo. Segundo Murphey (1998), o gluconato de cálcio estimula a contração da musculatura lisa, promovendo efeito peristáltico. $\mathrm{O}$ maior volume da solução eletrolítica infundido por via enteral diretamente no estômago também pode ter colaborado para o mencionado aumento, pois, como citaram Argenzio et al. (1974) e Fenger et al. (2000), o reflexo gastrocólico envolve o estímulo da motilidade colônica em resposta à distensão gástrica. Corroborando estes resultados, Avanza (2007) relatou aumento da motilidade intestinal após a administração de soluções eletrolíticas por via enteral por meio de sonda nasogástrica, na dose de $15 \mathrm{~mL} \mathrm{Kg}^{-1} \mathrm{~h}^{-1}$, e considerou o reflexo gastrocólico como a possível causa. Além disso, a solução enteral possuiu maior osmolaridade ou tonicidade. Soluções enterais de maior osmolaridade podem estimular a secreção de fluido para o lúmen intestinal devido à hipertonicidade intraluminal (MacGiness et al., 1996). 
Efeitos laxativos do polietilenoglicol...

Tabela 1. Medianas da auscultação dos quadrantes abdominais de equinos tratados em delineamento cross-over 5X5 com PEG, PEG+RL, SIPE, SIPE+RL e NaCl 0,9\%, nos tempos pré (T0h), trans (T6h e T12h) e pós (T24h e T48h) tratamento

\begin{tabular}{|c|c|c|c|c|c|}
\hline \multirow[t]{2}{*}{ Tratamento } & \multicolumn{5}{|c|}{ Tempo } \\
\hline & T0h & T6h & $\mathrm{T} 12 \mathrm{~h}$ & T24h & T48h \\
\hline & \multicolumn{5}{|c|}{ Auscultação do quadrante dorsal direito } \\
\hline PEG & 2 & 1 & 1 & 2 & 2 \\
\hline PEG+RL & 2 & 2 & 2 & 2 & 2 \\
\hline SIPE & 2 & 2 & 2 & 2 & 2 \\
\hline SIPE+RL & 2 & 2 & 2 & 2 & 2 \\
\hline \multirow[t]{2}{*}{$\mathrm{NaCl} 0,9 \%$} & 2 & 1 & 2 & 2 & 2 \\
\hline & \multicolumn{5}{|c|}{ Auscultação do quadrante ventral direito } \\
\hline PEG & 2 & 1 & 1 & 2 & 2 \\
\hline PEG+RL & 2 & 2 & 2 & 2 & 2 \\
\hline SIPE & 2 & 2 & 2 & 2 & 2 \\
\hline SIPE+RL & 2 & 2 & 2 & 2 & 2 \\
\hline \multirow[t]{2}{*}{$\mathrm{NaCl} 0,9 \%$} & 2 & 1 & 2 & 2 & 2 \\
\hline & \multicolumn{5}{|c|}{ Auscultação do quadrante ventral esquerdo } \\
\hline PEG & 2 & 1 & 1 & 2 & 2 \\
\hline PEG+RL & 2 & 2 & 2 & 2 & 2 \\
\hline SIPE & 2 & 2 & 3 & 2 & 2 \\
\hline SIPE+RL & 2 & 2 & 2 & 2 & 2 \\
\hline \multirow[t]{2}{*}{$\mathrm{NaCl} 0,9 \%$} & 2 & 2 & 2 & 2 & 2 \\
\hline & \multicolumn{5}{|c|}{ Auscultação do quadrante dorsal esquerdo } \\
\hline PEG & 2 & 1 & 1 & 2 & 2 \\
\hline PEG+RL & 2 & 2 & 2 & 2 & 2 \\
\hline SIPE & 2 & 2 & 3 & 2 & 2 \\
\hline SIPE+RL & 2 & 2 & 2 & 2 & 2 \\
\hline $\mathrm{NaCl} 0,9 \%$ & 2 & 2 & 2 & 2 & 2 \\
\hline
\end{tabular}

Escores da motilidade intestinal: ausente $=0$; diminuída $=1$; sem alteração $=2$; aumentada $=3$; PEG polietilenoglicol 3350 por via enteral (VE); PEG+RL - polietilenoglicol 3350, VE, associado ao Ringer lactato por via intravenosa (IV); SIPE - solução isotônica poliônica enteral, VE; SIPE + RL - solução isotônica poliônica enteral, VE, associada ao Ringer lactato IV; $\mathrm{NaCl}$ 0,9\% - solução de cloreto de sódio 0,9\%, IV.

Como não houve hipomotilidade intestinal nos tratamentos PEG+RL, SIPE e SIPE+RL no referido período (Tab. 1), é possível que o cálcio contido nas soluções administradas nesses grupos tenha contribuído para a manutenção da motilidade intestinal, principalmente o gluconato de cálcio, nos grupos em que foi utilizada a solução enteral, pois, no SIPE, ocorreu aumento da motilidade intestinal no T12h nos quadrantes dorsal e ventral do abdômen esquerdo. Segundo Murphey (1998), o gluconato de cálcio estimula a contração da musculatura lisa, promovendo efeito peristáltico. O maior volume da solução eletrolítica infundido por via enteral diretamente no estômago também pode ter colaborado para o mencionado aumento, pois, como citaram
Argenzio et al. (1974) e Fenger et al. (2000), o reflexo gastrocólico envolve o estímulo da motilidade colônica em resposta à distensão gástrica. Corroborando estes resultados, Avanza (2007) relatou aumento da motilidade intestinal após a administração de soluções eletrolíticas por via enteral por meio de sonda nasogástrica, na dose de $15 \mathrm{~mL} \mathrm{Kg}^{-1} \mathrm{~h}^{-1}$, e considerou o reflexo gastrocólico como a possível causa. Além disso, a solução enteral possuiu maior osmolaridade ou tonicidade. Soluções enterais de maior osmolaridade podem estimular a secreção de fluido para o lúmen intestinal devido à hipertonicidade intraluminal (MacGiness et al., 1996). 
Tabela 2. Frequências das defecações, valores médios das frequências das defecações e aspecto das fezes de equinos tratados em delineamento cross-over $5 \mathrm{X} 5$ com PEG, PEG+RL, SIPE, SIPE+RL e NaCl 0,9\%, em intervalos de seis horas durante um período de 48 horas

\begin{tabular}{|c|c|c|c|c|c|c|c|c|}
\hline \multirow[t]{2}{*}{ Tratamento } & \multicolumn{8}{|c|}{ Intervalo de tratamento $(\mathrm{h})$} \\
\hline & $0-6$ & $6-12$ & $12-18$ & $18-24$ & $24-30$ & $30-36$ & $36-42$ & $42-48$ \\
\hline \multicolumn{9}{|c|}{ Frequências das defecações e aspecto das fezes } \\
\hline PEG & $\mathbf{1}^{*}(1)^{* * *}$ & $6(1)$ & $6(1)$ & $6(1)$ & $8(1)$ & $10(1)$ & $15(1)$ & $10(1)$ \\
\hline PEG+RL & $\mathbf{0}$ & $8(1)$ & 7(1) $1(2)$ & 11(1) 2(2) & $\mathbf{9}(1)$ & $16(1)$ & 14(1) & 14(1) \\
\hline SIPE & $\mathbf{0}$ & 11(1) $4(2)$ & $4(1) 6(2) 3(3)$ & $\mathbf{3}(1) \mathbf{4}(2)$ & $\begin{array}{c}\mathbf{4}(1) \mathbf{4}(2) \\
8: 4(2), 4(1)\end{array}$ & $15(1)$ & 19(1) & $15(1)$ \\
\hline SIPE+RL & $3(1)$ & $3(1)$ & 11(1) & 6(1) 2(2) & 6(1) $1(2)$ & 12(1) & $12(1)$ & $12(1)$ \\
\hline $\mathrm{NaCl} 0,9 \%$ & $4(1)$ & $6(1)$ & $\mathbf{1 3}(1)$ & 9(1) 2(2) & 11(1) & $16(1)$ & 14(1) & $15(1)$ \\
\hline
\end{tabular}

* número de defecações; ${ }^{* *}$ escore do aspecto das fezes: $0=$ ressequidas; $1=$ normais; $2=$ pastosas; $3=$ aquosas (diarreicas); PEG - polietilenoglicol 3350 por via enteral (VE); PEG+RL - polietilenoglicol 3350, VE, associado ao Ringer lactato por via intravenosa (IV); SIPE - solução isotônica poliônica enteral, VE; SIPE + RL - solução isotônica poliônica enteral, VE, associada ao Ringer lactato, IV; $\mathrm{NaCl}$ 0,9\% - solução de cloreto de sódio 0,9\%, IV.

No grupo que recebeu solução enteral associada à hidratação intravenosa (SIPE+RL), o efeito sobre as fezes foi verificado após o final da fase de tratamento, quando se observou a eliminação de fezes pastosas nos tempos $18-24 \mathrm{~h}$ e $24-30 \mathrm{~h}$. Esse achado demonstrou que o volume da solução eletrolítica utilizado no presente estudo $\left(15 \mathrm{~mL} \mathrm{~kg}^{-1} \mathrm{~h}^{-1}\right)$ foi eficaz em amolecer as fezes. Porém, quando esse volume total foi administrado por via enteral (SIPE), o efeito foi mais acentuado do que quando se fracionou essa quantidade, metade por via enteral e a outra por via intravenosa, demonstrando que a via enteral foi mais efetiva do que a intravenosa em ocasionar o amolecimento das fezes (Tab. 2).
$\mathrm{O}$ tratamento PEG+RL também foi efetivo em ocasionar o amolecimento das fezes entre a $12^{\mathrm{a}} \mathrm{e}$ a $24^{\mathrm{a}}$ hora. É provável que o resultado observado tenha sido decorrente da ação do PEG neste tratamento. Lopes (2002) também relatou aumento significativo do teor de água nas fezes de equinos tratados durante seis horas com Ringer lactato IV associado a laxativo (sulfato de magnésio) por via enteral. Foi detectado aumento na frequência da defecação no referido grupo em relação aos demais apenas no tempo 18-24h. Mas, como já tinham passadas aproximadamente 12 horas do retorno da dieta, é possível que o aumento do número de defecações tenha sido também influenciado por esse evento.

Tabela 3. Valores médios e desvios-padrão da umidade das fezes (\%) de equinos tratados em delineamento cross-over 5X5 com PEG, PEG+RL, SIPE, SIPE+RL e NaCl 0,9\%, nos tempos pré (T0h), inter (T6h e T12h) e pós (T24h e T48h) tratamento

\begin{tabular}{lccccc}
\hline \multirow{2}{*}{ Tratamento } & \multicolumn{5}{c}{ Tempo } \\
\cline { 2 - 6 } & T0h & T6h & T12h & T24h & T48h \\
\hline PEG & $71,36 \pm 0,92 \mathrm{Aa}$ & $66,57 \pm 0,71 \mathrm{Aa}$ & $68,16 \pm 0,64 \mathrm{Aa}$ & $69,74 \pm 0,60 \mathrm{Aa}$ & $68,46 \pm 0,65 \mathrm{Aa}$ \\
PEG+RL & $70,92 \pm 0,76 \mathrm{Aa}$ & $69,43 \pm 0,88 \mathrm{Aa}$ & $72,31 \pm 1,24 \mathrm{Aa}$ & $71,07 \pm 0,90 \mathrm{Aa}$ & $68,94 \pm 0,95 \mathrm{Aa}$ \\
SIPE & $72,20 \pm 1,04 \mathrm{Aab}$ & $71,37 \pm 0,92 \mathrm{Aab}$ & $78,50 \pm 1,68 \mathrm{Aa}$ & $72,83 \pm 0,88 \mathrm{Aa}$ & $69,44 \pm 0,72 \mathrm{Ab}$ \\
SIPE+RL & $72,05 \pm 0,79 \mathrm{Aa}$ & $72,30 \pm 1,20 \mathrm{Aa}$ & $68,24 \pm 0,90 \mathrm{Aa}$ & $71,67 \pm 0,81 \mathrm{Aa}$ & $70,75 \pm 0,78 \mathrm{Aa}$ \\
NaCl 0,9\% & $69,90 \pm 0,78 \mathrm{Aa}$ & $71,70 \pm 0,85 \mathrm{Aa}$ & $70,00 \pm 0,96 \mathrm{Aa}$ & $71,81 \pm 0,95 \mathrm{Aa}$ & $70,43 \pm 0,79 \mathrm{Aa}$ \\
\hline
\end{tabular}

Valores médios seguidos por letras distintas minúsculas na linha diferem entre si $(p<0,05)$ pelo teste Tukey. Não foi observada diferença entre tratamentos $(\mathrm{P}>0,05)$. PEG - polietilenoglicol 3350 por via enteral (VE); PEG+RL polietilenoglicol 3350, VE, associado ao Ringer lactato por via intravenosa (IV); SIPE - solução isotônica poliônica enteral, VE; SIPE + RL - solução isotônica poliônica enteral, VE, associada ao Ringer lactato, IV; NaCl 0,9\% solução de cloreto de sódio $0,9 \%$, IV. 
No grupo $\mathrm{NaCl} 0,9 \%$, fezes pastosas foram observadas em apenas um animal no tempo 1824h (Tab. 2). Aumento no número de defecações também foi observado no tempo 12-18h, demonstrando resultado semelhante ao SIPE e próximo ao do SIPE+RL.

No grupo de animais do PEG, não foram observadas alterações na frequência de defecação (Tab. 2) nem na umidade das fezes (Tab. 3). Entretanto, deve-se ressaltar que dois animais apresentaram duas defecações com fezes parcialmente com características normais entremeadas a partes com aspecto pastoso ("semipastosas") entre a $30^{a}$ e a $36^{a}$ hora, ou seja, houve discreta alteração no aspecto das fezes, sinalizando que houve exígua ação desse fármaco. Considerando-se que o intestino do equino adulto é de tamanho bem maior que o do adulto humano, para que um laxativo utilizado neste possa exercer efeito em equinos, a dose deve aumentar aproximadamente cinco vezes (Freeman et al., 1992). No presente estudo, essa recomendação foi executada, porém o efeito laxativo dessa substância pode ser considerado de pouco significado clínico. Contudo, mesmo com o pobre resultado observado após a administração do PEG, pode-se pressupor que uma dose maior, ou uma segunda dose de $1,5 \mathrm{~g}$ $\mathrm{Kg}^{-1}$ após intervalo de doze horas, poderia proporcionar um melhor efeito laxativo dessa substância, o que requer, portanto, estudos subsequentes em busca destas questões.

Tabela 4. Valores médios do tempo médio de retenção (TMR) (h) e taxa de passagem (TxP) $\left(\% \mathrm{~h}^{-1}\right)$ da ingesta no intestino grosso (ig) durante $144 \mathrm{~h}$, em equinos tratados em delineamento cross-over $5 \mathrm{X} 5 \mathrm{com}$ PEG, PEG+RL, SIPE, SIPE+RL e NaCl 0,9\%

\begin{tabular}{lcc}
\multicolumn{1}{c}{ Tratamentos } & TMRig $(\mathrm{h})$ & TxPig $\left(\% \mathrm{~h}^{-1}\right)$ \\
\hline PEG & $62,14 \mathrm{AB}$ & $3,47 \mathrm{AB}$ \\
PEG+RL & $69,01 \mathrm{~A}$ & $3,16 \mathrm{~B}$ \\
SIPE & $53,22 \mathrm{~B}$ & $4,17 \mathrm{~A}$ \\
$\mathrm{SIPE}+\mathrm{RL}$ & $57,92 \mathrm{AB}$ & $3,72 \mathrm{AB}$ \\
$\mathrm{NaCl} \mathrm{0,9 \%}$ & $62,49 \mathrm{AB}$ & $3,49 \mathrm{AB}$
\end{tabular}

Valores médios na mesma coluna seguidos por letras distintas diferem entre si $(\mathrm{P}<0,05)$ pelo teste Tukey; PEG polietilenoglicol 3350 por via enteral (VE); PEG+RL - polietilenoglicol 3350, VE, associado ao Ringer lactato por via intravenosa (IV); SIPE - solução isotônica poliônica enteral, VE; SIPE + RL - solução isotônica poliônica enteral, VE, associada ao Ringer lactato, IV; $\mathrm{NaCl} \mathrm{0,9 \%} \mathrm{-} \mathrm{solução} \mathrm{de} \mathrm{cloreto} \mathrm{de} \mathrm{sódio} \mathrm{0,9 \% ,} \mathrm{IV.}$

O tratamento SIPE foi o que apresentou o menor tempo de retenção no intestino grosso, portanto maior taxa de passagem pelo ceco e cólons (Tab. 4), ou seja, entre os tratamentos estudados, foi o que mais abreviou o tempo de trânsito da ingesta no intestino grosso. Entretanto, esse resultado foi significativo $(\mathrm{P}<0,05)$ apenas em relação ao PEG+RL. Lopes (2002) detectou menor TMRig em equinos fistulados tratados com solução eletrolítica isotônica por via enteral na dose de $5 \mathrm{~mL} \mathrm{~kg}{ }^{-1}$ durante $12 \mathrm{~h}$, quando comparado a outros quatro tratamentos: sulfato de magnésio, sulfato de sódio, Ringer lactato IV, e água. Ribeiro Filho (2003) registrou 100\% de fezes diarreicas em equinos com compactação induzida do cólon maior tratados com solução eletrolítica isotônica enteral na dose de $8 \mathrm{~mL} \mathrm{~kg}^{-1}$ $48 \mathrm{~h}^{-1}$. Os resultados desses autores foram confirmados com os do presente ensaio.
Quando se avaliou o conjunto dos parâmetros mensurados, motilidade intestinal, frequência de defecação, aspecto e teor de umidade das fezes, TMRig e TxPig (Tab. 1, 2, 3 e 4), observou-se que o tratamento SIPE foi o que ocasionou os efeitos laxativos mais acentuados. Pelo fato de a hidratação enteral com soluções eletrolíticas apresentar ação laxativa significativa, além de corrigir os desequilíbrios hidroeletrolíticos e ácido-base, usualmente detectados em pacientes que necessitam desse tipo de terapia, ela pode ser utilizada em substituição ao uso clássico de laxantes associados à hidratação intravenosa, afirmação essa que pode ser corroborada pelos resultados obtidos por Freeman et al. (1992), Lopes (2002) e Ribeiro Filho (2003). 


\section{CONCLUSÕES}

O tratamento PEG ocasiona um efeito laxativo brando, por provocar apenas um discreto amolecimento das fezes, tornando-as semipastosas. Os tratamentos PEG+RL, SIPE+RL e $\mathrm{NaCl} 0,9 \%$ provocam amolecimento das fezes e aumento da frequência de defecações, portanto são um pouco mais efetivos que o PEG. O tratamento com a solução isotônica poliônica enteral (SIPE) causa aumento na frequência de defecações e amolecimento das fezes, acelera a TxPig, ou seja, diminui o TMRig, demonstrando ser, entre os tratamentos estudados, o melhor em proporcionar efeito laxativo em equinos.

\section{AGRADECIMENTOS}

À FAPEMIG (Fundação de Amparo à Pesquisa do Estado de Minas Gerais).

\section{REFERÊNCIAS}

ARGÊNZIO, R.A.; LOWE, J.E.; PICKARD, D.W. et al. Ingesta passage and water exchange in the equine large intestine. Am. J. Physiol., v.226, p.1035-1042, 1974

AVANZA, M.F.B. Soluções eletrolíticas associadas ou não a glicose, maltodextrina e sulfato de magnésio administrada por via enteral em equinos hígidos $e$ desidratados experimentalmente. 2007, 68f. Dissertação (Mestrado em Medicina Veterinária) Departamento de Veterinária, Universidade Federal de Viçosa, Viçosa, MG.

CLARK, E.S.; BECHT, J.L. Clinical pharmacology of the gastrointestinal tract. Vet. Clin. North Am. Equine Pract., v.3, p.101-122, 1987.

CORAZZIARI, E.; BADIALI, D.; BAZZOCCHIB, G. et al. Small volume isosmotic polyethylene glycol electrolyte balanced solution (PMF-100) in treatment of chronic nonorganic constipation. Digest. Dis. Sci., v.41, p.1636-1642, 1996.

DABAREINER, R.M.; WHITE, N.A. Large colon impaction in horses: 147 cases (1985-1991). J. Am. Vet. Med. Assoc., v.206, p.679-85, 1995.

ELLIS, W.C.; MATIS, J.H.; HILL, T.M. et al. Methodology for estimating digestion and passage kinects of forages. In: FATHEY, J.R.G.C. (Ed.). Forage quality. Evaluation and utilization. Winsconsin: American Society of Agronomy, 1994. p.450-493.
FENGER, C.K.; BERTONE, A.L.; BERTONE, J.J. Motilidade gastrintestinal e íleo paralítico. In: REED, S.M.; BAYLY, W.M. Princípios farmacológicos. Medicina Interna Equina. Rio de janeiro: Guanabara Koogan, 2000. p.178-185.

FREEMAN, D.E.; FERRANTE, P.I.; PALMER, J.E. Comparison of the effects of antragastric infusions of equal volumes of water, dioctyl sodium sulfoccinato, and magnesium sulfate on fecal composition and output in clinically normal horses. Am. J. Vet. Res., v.53, p.1347-1353, 1992

HAMMER, H.F.; SANTANA, C.A.; SCHILLER, L.R. et al. Studies of osmotic diarrhea induced in normal subjects by ingestion of polyethylene glycol and lactulose. J. Clin. Invest., v.84, p.1056-1062, 1989.

LOPES, M.A.F. Hydration of colonic ingest and feces in horses fed large grain meals or treated with enteral fluid therapy, saline cathartics and intravenous fluid therapy. 2002. 147f. Tese (Doctorade in Philosophy of Science Veterinary Medical) - Faculty of Virginia Polytechnic Institute, Blacksburg, Virginia.

MACORIS, D.G. Trânsito intestinal em equinos: efeito dos tratamentos com flunixin meglumina, dipirona-hioscina e óleo mineral. 1989. 28f. Tese (Doutorado em Medicina Veterinária) - Universidade Estadual de São Paulo, Botucatu, SP.

MacGINNESS, S.G.; MANSMANN, R.A.; BREUHAUS, B.A. Nasogastric electrolyte replacement in horses. Cont. Educ. Art., v.18, p.43-49, 1996.

MURPHEY, D.D. Manegement of adynamic ileus. In: REED, S.M.; BAYLY, W.M. Equine internal medicine. Philadelphia: Saunders, 1998. p.694-700.

RIBEIRO FILHO, J.D. Tratamento da compactação experimental do cólon maior de equinos com sene, fluidoterapia enteral e parenteral. 2003, 130f. Tese (Doutorado em Ciência Animal) - Escola de Veterinária, Universidade Federal de Minas Gerais, Belo Horizonte, MG.

SISTEMA para análises estatísticas - SAEG. Versão 9.1. Viçosa: UFV, Fundação Arthur Bernardes, 2007. $301 \mathrm{p}$.

SULLINS, K.E. Disease of the large colon. In: WHITE, N.A. (Ed). Equine acute abdomen. Philadelphia: Lea \& Febiger, 1990, cap.10, p.375-391.

THOMASSIAN, A. (Ed.). Enfermidades dos cavalos. 4.ed. São Paulo: Varela, 2005. 573p.

ÚDEN, P.; COLUCCI, P.E.; VAN SOEST, P.J. Investigation chromium, cerium and cobalt as markers in ingesta. Rate of passage studies. J. Sci. Food Agri., v.31, p.625-632, 1980. 